\title{
Modelo Experimental de Reestenose Intrastent em Artérias Coronárias de Suínos: Efeito do Implante de Stent Sobredimensionado
}

\author{
José Casco Raudales', Alcides J. Zago', Paulo R. Centeno Rodrigues², Beatriz G. Kosachenco², \\ Joice C. Benetti ${ }^{1}$, Diovana Dallarosa ${ }^{1}$, Cristina Dreyer ${ }^{2}$, Juliana G. Ferst ${ }^{1}$, Marco A. Bortolini ${ }^{1}$, \\ Sandro P. Ramos' ${ }^{2}$, Alexandre do Canto Zago ${ }^{1}$
}

\section{RESUMO}

Introdução: Há múltiplos modelos experimentais em animais, entretanto o modelo suíno é o que apresenta características anatômicas e funcionais mais próximas às humanas. Assim sendo, este trabalho foi realizado com o objetivo de desenvolver e implementar um protocolo experimental de indução de hiperproliferação neointimal em suínos, visando à criação de técnicas de lesão vascular simulando a reestenose. Método: De agosto de 2006 a março de 2009, 69 suínos jovens da raça Large White foram submetidos a cinecoronariografia seguida de lesão vascular com implante de 102 stents sobredimensionados, guiados por ultrassom intracoronário. Em 28 dias foi realizado reestudo com nova cinecoronariografia e ultrassom intracoronário. Resultados: $\mathrm{O}$ diâmetro luminal mínimo e a área luminal mínima imediatamente após o implante de stent no grupo stent sobredimensionado foram maiores em comparação ao grupo controle $(3,5 \pm 0,3 \mathrm{~mm}$ vs. $3 \pm 0,2 \mathrm{~mm}$, $\mathrm{P}<0,0001$ e $40,7 \pm 0,3 \mathrm{~mm}^{2}$ vs. $\left.30,2 \pm 0,2 \mathrm{~mm}^{2}, \mathrm{P}<0,0001\right)$. A taxa de reestenose binária no grupo submetido a implante de stent sobredimensionado foi de $92 \%$ (69/75 stents), enquanto no grupo controle foi de $12 \%$ (3/25 stents), com diferença estatisticamente significante $(P<0,0001)$. O volume de hiperplasia neointimal foi maior no grupo submetido a implante de stent sobredimensionado, em comparação ao grupo controle $\left(5,9 \pm 0,8 \mathrm{~mm}^{3} / \mathrm{mm}\right.$ de stent vs. $1,8 \pm 0,7 \mathrm{~mm} 3 / \mathrm{mm}$ de stent; $P<0,0001)$. Conclusão: $O$ modelo experimental proposto de indução de proliferação neointimal em suínos é eficaz na indução de hiperplasia intrastent, portanto pode ser utilizado tanto para o estudo dos mecanismos fisiopatológicos da reestenose intrastent quanto para aplicações terapêuticas, como testes de novos
ABSTRACT

Experimental Model of In-Stent Restenosis in Swine Coronary Arteries: Effect of Oversized Stent Deployment

Background: There are several experimental animal models, but, the swine model is the most similar to human anatomic and physiologic characteristics. Therefore, this study was carried out to develop and implement an experimental protocol of vascular neointimal hyperplasia induction in swine, aiming at creating vascular injury techniques simulating restenosis. Method: From August 2006 to March 2009, 69 young Large White swine underwent coronary angiography followed by vascular injury and implantation of 102 oversized stents guided by intravascular ultrasound. After 28 days a new coronary angiography and intravascular ultrasound was performed. Results: The minimal luminal diameter and the minimal luminal area immediately after the stent deployment in the group treated with an oversized stent were significantly higher when compared to the control group $(3.5 \pm 0.3 \mathrm{~mm}$ vs. $3 \pm 0.2 \mathrm{~mm}, \mathrm{P}<0.0001$ and $40.7 \pm 0.3 \mathrm{~mm}^{2}$ vs. $\left.30.2 \pm 0.2 \mathrm{~mm}^{2}, \mathrm{P}<0.0001\right)$. The binary restenosis rate in the group treated with an oversized stent was $92 \%$ (69/75 stents), whereas it was $12 \%(3 / 25$ stents) in the control group, with a statistically significant difference $(P<0.0001)$. The neointimal hyperplasia volume was significantly higher in the group treated with an oversized stent in comparison to the control group $(5.9 \pm$ $0.8 \mathrm{~mm}^{3} /$ stent $\mathrm{mm}$ vs. $1.8 \pm 0.7 \mathrm{~mm}^{3} /$ stent $\left.\mathrm{mm}, \mathrm{P}<0.0001\right)$. Conclusion: The proposed experimental model of neointimal proliferation induction in swine is effective in inducing instent hyperplasia, and therefore it may be used for the

1 Centro de Pesquisa Cardiovascular da Universidade Luterana do Brasil/ULBRA - Canoas, RS, Brasil.

2 Hospital Veterinário da Universidade Luterana do Brasil/ULBRA Canoas, RS, Brasil.

Correspondência: Alexandre C. Zago. Centro de Pesquisa Cardiovascular da Universidade Luterana do Brasil/ULBRA - Av. Farroupilha, 8001 - prédio 22 - 5o andar - Canoas, RS, Brasil - CEP 92425-900

E-mail: zagoac@uol.com.br

Recebido em: 16/12/2009 - Aceito em: 7/3/2010 
Raudales JC, et al. Modelo Experimental de Reestenose Intrastent em Artérias Coronárias de Suínos: Efeito do Implante de Stent Sobredimensionado. Rev Bras Cardiol Invasiva. 2010;18(1):55-61.

fármacos, novos dispositivos e novos stents farmacológicos para prevenção e tratamento da reestenose intrastent.

DESCRITORES: Angioplastia transluminal percutânea coronária. Modelos animais. Suíno. Reestenose coronária. Stents.

D os múltiplos modelos experimentais em animais, seja canino, murino, ovino, leporino ou suíno, o modelo suíno é o que apresenta características anatômicas e funcionais mais próximas às humanas. Além disso, inúmeros estudos experimentais têm demonstrado que a resposta hiperproliferativa, caracterizada pela formação de neoíntima decorrente de lesão do endotélio vascular em suínos é histologicamente similar àquela observada em humanos. ${ }^{1-7}$

O modelo suíno foi descrito originalmente por Schwartz et al. ${ }^{5}$ utilizando balões de angioplastia para produzir lesão na íntima vascular das artérias coronárias sadias de suínos jovens; posteriormente foram utilizados stents metálicos sobredimensionados com o mesmo objetivo. ${ }^{4,6-8}$ Do ponto de vista anatômico, as artérias coronárias suínas são muito semelhantes às coronárias humanas, no entanto apresentam menor propensão ao desenvolvimento de circulação colateral. ${ }^{4}$ O tronco de coronária esquerda bifurca, originando as artérias descendente anterior esquerda e circunflexa, as quais têm diâmetro similar ao das coronárias humanas $(2 \mathrm{~mm}$ a $4 \mathrm{~mm})$. A artéria circunflexa dá origem a até 3 ramos marginais, enquanto a descendente anterior irriga o septo através de 4 a 6 ramos septais. A artéria coronária direita dos suínos é tão calibrosa quanto a humana, porém fornece ramos para a irrigação da superfície posterior do coração em menor frequência que a de humanos, em que a coronária direita é dominante em $80 \%$ dos casos. $^{4}$

A justificativa desse modelo está baseada no fato de que os testes de novos dispositivos cardiovasculares intervencionistas, como os stents, precisam de um modelo animal adequado para avaliar, em curto prazo, a segurança dos protótipos, em termos de biocompatibilidade, taxas de sucesso do procedimento e eventos cardíacos adversos, bem como sua eficácia, mediante a incidência de reestenose angiográfica. O Food and Drug Administration (FDA) tem diretrizes que exigem a avaliação dos dispositivos de angioplastia coronária em modelo animal, prévio à aprovação para pesquisa clínica. ${ }^{9}$

Outra justificativa importante proposta por Schwartz et al. ${ }^{7}$ é a necessidade de elucidar e entender melhor os mecanismos da reestenose intrastent humana. Assim sendo, modelos pré-clínicos permitem metodologias de estudo direcionadas e o desenvolvimento de novas estratégias de revascularização. study of the pathophysiologic mechanisms of in-stent restenosis as well as for therapeutic purposes, such as the evaluations of new drugs, new devices and new drug-eluting stents for the prevention and treatment of in-stent restenosis.

KEY-WORDS: Angioplasty, transluminal, percutaneous coronary. Models, animal. Swine. Coronary restenosis. Stents.

Além disso, não há no momento um dispositivo ou técnica considerada padrão de referência para o tratamento da reestenose intrastent, portanto esse modelo experimental pode contribuir para desenvolver e avaliar os resultados pré-clínicos de novas estratégias de tratamento da reestenose intrastent.

O objetivo deste trabalho é desenvolver e implementar um protocolo experimental de indução de hiperproliferação neointimal em artérias coronárias de suínos, mediante a utilização de técnicas de lesão vascular que induzem reestenose intrastent.

\section{MÉTODO}

\section{Estrutura física}

Para realizar as angiografias coronárias, os implantes de stents e os exames de ultrassom intracoronário, foi treinada uma equipe multidisciplinar composta por médicos, veterinários, enfermeiras e técnicos e montada uma Unidade de Hemodinâmica Experimental com a mesma estrutura de uma unidade para uso humano, localizada dentro do bloco cirúrgico do Hospital Veterinário da Universidade Luterana do Brasil (ULBRA) (Figura 1). O aparelho de cineangiografia utilizado foi o modelo Angio Diagnost 5 da marca Philips e as imagens foram digitalizadas pelo sistema Digital Cardiac Imaging (DCl), sendo posteriormente

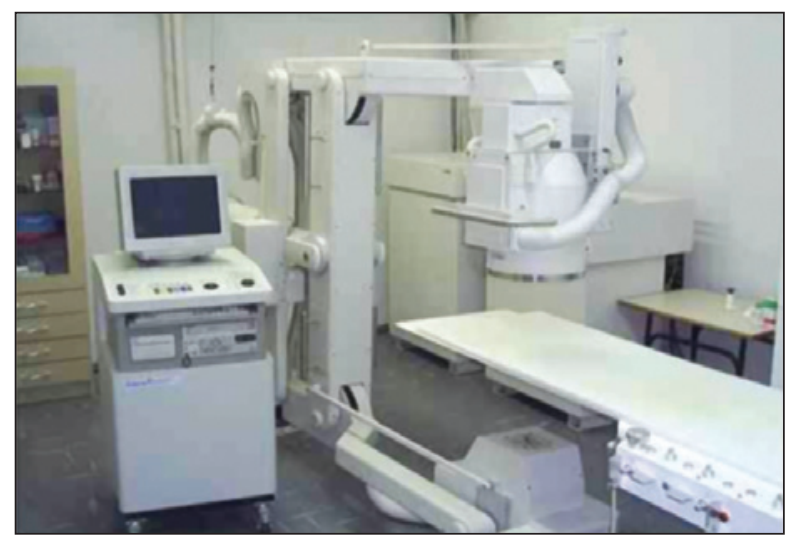

Figura 1 - Equipamentos do Laboratório de Hemodinâmica Experimental do Centro de Pesquisa Cardiovascular da Universidade Luterana do Brasil/ULBRA (Canoas, RS). 
Raudales JC, et al. Modelo Experimental de Reestenose Intrastent em Artérias Coronárias de Suínos: Efeito do Implante de Stent Sobredimensionado. Rev Bras Cardiol Invasiva. 2010;18(1):55-61.

gravadas e armazenadas em discos compactos (CDM 3300 recorder). O aparelho de ultrassom intracoronário utilizado foi o Oracle In-Vision Imaging System V 3.3.1 (EndoSonics Co., Estados Unidos) com histologia virtual e as imagens foram obtidas por meio dos cateteres Eagle Eye ${ }^{\circledR}$ (Volcano Therapeutics Inc., Estados Unidos).

\section{Suínos}

Foram utilizados suínos jovens da raça Large White, com peso inicial aproximado de $45 \mathrm{~kg}$ a $50 \mathrm{~kg}$. Em todos os procedimentos foram seguidos os protocolos de pesquisa animal conforme estabelecido pela Comissão de Ensino do Colégio Brasileiro de Experimentação Animal $(\mathrm{COBEA})^{10}$ e pelo Conselho Federal de Medicina Veterinária. ${ }^{11} \mathrm{O}$ projeto foi aprovado pelo Comitê de Ética em Pesquisa Humana e Animal da ULBRA e realizado no Laboratório de Hemodinâmica Experimental do Centro de Pesquisa Cardiovascular da ULBRA.

\section{Anestesia}

A medicação pré-anestésica foi realizada com atropina $0,044 \mathrm{mg} / \mathrm{kg} \mathrm{IM}$, acepromazina $0,1 \mathrm{mg} / \mathrm{kg} \mathrm{IM}$, midazolan $0,5 \mathrm{mg} / \mathrm{kg} \mathrm{IM}$ e azaperone $2 \mathrm{mg} / \mathrm{kg} \mathrm{IM}$. A indução anestésica foi realizada com uma associação de tiletamina/zolazepam $4 \mathrm{mg} / \mathrm{kg} \mathrm{EV}$, seguida de intubação traqueal e ventilação espontânea com oxigênio a $100 \%$. A manutenção da anestesia foi realizada com isoflurano, utilizando um vaporizador universal. A monitorização hemodinâmica incluiu controle das frequências cardíaca e respiratória, eletrocardiograma de superfície, oximetria de pulso e controle da temperatura corporal.

\section{Abordagem vascular e transoperatório}

A abordagem vascular foi realizada por meio de incisão cirúrgica por planos nas regiões inguinal ou cervical ventral, seguida de dissecção vascular das artérias femorais ou carótidas externas. A punção vascular foi realizada pela técnica de Seldinger modificada, seguida da inserção de uma bainha arterial 7 F. Concluído o acesso vascular, procedeu-se à administração sistêmica de heparina na dose de 100-150 Ul/kg $\mathrm{EV}^{4,7,12}$, com reforço a cada 30 minutos se necessário. Foi adotado o uso profilático do antiarrítmico amiodarona na dose de $150 \mathrm{mg}$ EV em dose única (o bretílio sugerido por Lowe et al. ${ }^{4}$ não é disponível no Brasil) e do vasodilatador coronário mononitrato de isossorbida $10 \mathrm{mg}$ intracoronário. A angiografia coronária foi realizada usando contraste hiperosmolar convencional (Pielograf; BerliMed, Espanha). As artérias coronárias foram canuladas por meio de cateteres com curvas especiais (AR1, HS, MP e SR) em função das características anatômicas particulares do arco aórtico dos suínos. ${ }^{4}$ Os equipamentos utilizados e os procedimentos diagnósticos e terapêuticos realizados seguiram as mesmas normas e técnicas preconizadas em seres humanos, sem modificações expressivas. ${ }^{13,14}$

\section{Protocolo de lesão vascular}

A partir da angiografia, foi selecionado um segmento arterial com diâmetro de referência entre $2,5 \mathrm{~mm}$ e $3 \mathrm{~mm}$ nos segmentos proximal, médio ou distal das artérias circunflexa ou coronária direita. ${ }^{4,15} \mathrm{~A}$ artéria descendente anterior esquerda foi a menos utilizada por ser menos extensa e por apresentar perda significativa de calibre a partir do segmento médio. ${ }^{4}$

Para induzir a reestenose intrastent foi utilizada a técnica de implante de um stent com dimensão sobredimensionada para lesar a parede arterial $\left.\right|^{2,4,6-8}$, ou seja, foi implantado um stent com diâmetro final 1,2 ou 1,3 maior $^{2,4,6-8,16-18}$ em relação ao diâmetro de referência do vaso, conforme os dados obtidos pela angiografia quantitativa. A extensão do stent implantado foi de $18 \mathrm{~mm}$. Foram realizadas duas insuflações de 35 segundos de duração cada, a uma pressão de 16 atm a $20 \mathrm{~atm}$ com intervalos de 30 segundos entre as insuflações. O grupo controle foi submetido a implante de stent, mantendo-se uma relação 1:1 entre os diâmetros do vaso e da prótese para evitar lesão vascular exacerbada e reestenose.

Após angiografia de controle e avaliação do implante com ultrassom intracoronário, utilizado para assegurar a expansão adequada do stent e a aposição das hastes na parede da artéria coronária e, também, para o cálculo das áreas e do volume da neoíntima, o cateter-guia e a bainha arterial foram retirados, a arteriotomia foi suturada e a dermorrafia foi realizada com sutura intradérmica.

As medicações utilizadas no pós-operatório, em doses únicas diárias, foram cetoprofeno $3 \mathrm{mg} / \mathrm{kg} \mathrm{VO}$ por 3 dias, oxitetraciclina $25 \mathrm{mg} / \mathrm{kg}$ VO por 8 dias e tiamulin $8,8 \mathrm{mg} / \mathrm{kg} \mathrm{VO}$ por 8 dias. Antibioticoterapia profilática foi realizada para evitar infecção da ferida operatória (considerada procedimento padrão do laboratório). ${ }^{7,19}$ Foi administrado ácido acetilsalicílico $100 \mathrm{mg} \mathrm{VO} /$ dia 2 dias antes e indefinidamente após o procedimento (a literatura sugere $81 \mathrm{mg} / \mathrm{dia}$ a $325 \mathrm{mg} /$ dia indefinidamente) $)^{7,19}$ e clopidogrel na dose inicial de $37,5 \mathrm{mg} \mathrm{VO} / \mathrm{dia}$, o qual foi aumentado para $75 \mathrm{mg} \mathrm{VO/}$ dia, conforme o ganho ponderal aproximava-se dos $70 \mathrm{~kg}$, e mantido durante pelo menos 3 meses. $^{7}$

\section{Seguimento clínico}

O tempo de observação dos suínos após a aplicação do protocolo de lesão vascular foi de 28 dias, que corresponde ao período suficiente para o desenvolvimento pleno da neoíntima intrastent, ou seja, no segmento arterial submetido a indução de lesão vascular. Assim sendo, em 28 dias após a lesão vascular, os suínos foram submetidos a cinecoronariografia com análise quantitativa, mantendo as mesmas projeções angiográficas e estudo com ultrassom intracoronário para avaliar o grau de proliferação neointimal e o porcentual de obstrução intrastent decorrente da neoíntima. 


\section{Análise estatística}

As variáveis categóricas foram expressas como frequências e porcentagens e comparadas pelo teste de qui-quadrado; as variáveis contínuas foram expressas como médias e desvio padrão e comparadas pelo teste $t$ de Student. $\mathrm{O}$ valor de $\mathrm{P}$ foi considerado significativo quando $\leq 0,05$.

\section{RESULTADOS}

De agosto de 2006 a março de 2009, 69 suínos foram submetidos a intervenção coronária percutânea no Laboratório de Hemodinâmica Experimental da ULBRA. Em 62 deles foram implantados 102 stents e realizados 202 exames de ultrassom intracoronário, com média de 1,7 stent por suíno. A taxa de sucesso dos implantes de stent foi de $98 \%$, havendo 2 casos de migração do stent, que se soltou do balão durante o procedimento e foi implantado mais distalmente, ultrapassando a relação diâmetro do stent/diâmetro do vaso máxima permitida de 1,3:1. Dos 100 stents implantados com sucesso, 75 foram implantados com dimensão superestimada, ou seja, com relação 1,2 ou 1,3:1 (Grupo A). Os outros 25 stents foram implantados nos suínos do grupo controle, mantendo-se relação 1:1 (Grupo B). A mortalidade foi observada em dois momentos: perioperatória (até 72 horas após o procedimento) e pós-operatória ( $>72$ horas a 28 dias após o procedimento). A mortalidade perioperatória foi de $10,1 \%$ (7 suínos), e os óbitos ocorreram no início do projeto (4 casos de septicemia, 2 casos de parada cardiorrespiratória e 1 caso de hipertermia maligna). Não ocorreram óbitos no período pós-operatório.

A angiografia quantitativa evidenciou diâmetros de referência dos vasos e diâmetros luminais mínimos pré-procedimento sem diferenças entre os grupos $A$ e B (Tabela 1). Houve diferença significativa entre o diâmetro luminal mínimo imediatamente após o implante $(3,5 \pm 0,3 \mathrm{~mm}$ e $3 \pm 0,2 \mathrm{~mm} ; \mathrm{P}<0,0001)$, assim como entre o diâmetro luminal mínimo no seguimento de 28 dias $(1,8 \pm 0,6 \mathrm{~mm}$ e 2,49 $\pm 0,6 \mathrm{~mm}$; $\mathrm{P}<0,0001)$. A perda tardia, observada na avaliação angiográfica aos 28 dias, foi significativamente maior no grupo de suínos tratados com stent sobredimensionado em comparação ao grupo controle $(1,7 \pm$ $0,32$ e $0,51 \pm 0,38 ; P<0,0001)$, assim como a reestenose binária $(92 \%$ vs. $12 \% ; \mathrm{P}<0,0001)$.

O ultrassom intracoronário mostrou não haver diferença entre a área luminal mínina antes do implante de stent nos grupos A e B $\left(27,4 \pm 4,3 \mathrm{~mm}^{2}\right.$ e 28,3 \pm $\left.3,9 \mathrm{~mm}^{2} ; \mathrm{P}=0,106\right)$ (Tabela 2). Houve diferença significativa entre a área luminal mínima imediatamente após o implante de stent nos grupos A e B $(40,7 \pm$ $0,3 \mathrm{~mm}^{2}$ e $\left.30,2 \pm 0,2 \mathrm{~mm}^{2} ; \mathrm{P}<0,0001\right)$, assim como entre a área luminal mínima no seguimento de 28 dias $\left(10,2 \pm 1,1 \mathrm{~mm}^{2}\right.$ vs. 19,5 $\left.\pm 1,1 \mathrm{~mm}^{2} ; \mathrm{P}<0,0001\right)$. O volume de hiperplasia neointimal foi significativamente maior no grupo de suínos tratados com stent sobredimensionado em comparação ao grupo controle $\left(5,9 \pm 0,8 \mathrm{~mm}^{3} / \mathrm{mm}\right.$ de stent vs. $1,8 \pm 0,7 \mathrm{~mm}^{3} / \mathrm{mm}$ de stent; $\mathrm{P}<0,0001)$ (Figura 2).

\section{DISCUSSÃO}

A hiperplasia neointimal intracoronária em suínos sadios pode ser causada mediante lesão do endotélio com dispositivo (balão ou stent) de diâmetro superestimado em torno de $20 \%$ a $30 \%{ }^{1-8,12,15-18}$, ou seja, uma relação de 1,2:1 ou 1,3:1 entre o diâmetro final do dispositivo e o diâmetro de referência do vaso. Em termos gerais, a resposta celular à lesão vascular nãoaterosclerótica iatrogênica é caracterizada por ser tempo-dependente e envolve diferentes mecanismos fisiopatológicos: trombose mural focal induzida pelo stent, evidente nas primeiras 24 horas; retração elástica e remodelamento geométrico vascular negativo do vaso; e presença de infiltrado celular inflamatório com pico aos 7 dias, mas ainda evidente aos 28 dias, com liberação de endotelinas, geração de trombina, ativação e migração de plaquetas, que, além de produzir vasoconstrição, também são responsáveis pela formação do trombo organizado de fibrina e pela liberação de fatores de crescimento, que por sua vez favorecem a migração e a proliferação de células musculares lisas, neutrófilos e macrófagos, assim como a produ-

TABELA 1

Dados da angiografia coronária quantitativa

\begin{tabular}{lccc}
\hline & $\begin{array}{c}\text { Grupo A } \\
(\mathbf{n = 7 5 )}\end{array}$ & $\begin{array}{c}\text { Grupo B } \\
(\mathbf{n = 2 5})\end{array}$ & $\mathbf{P}$ \\
\hline $\begin{array}{l}\text { Diâmetro de referência do vaso }(\mathrm{mm}) \\
\text { Diâmetro luminal mínimo }\end{array}$ & $2,8 \pm 0,4$ & $2,9 \pm 0,3$ & 0,718 \\
$\quad$ Pré-stent & $2,8 \pm 0,4$ & $2,9 \pm 0,3$ & 0,718 \\
Pós-stent & $3,5 \pm 0,3$ & $3 \pm 0,2$ & $<0,0001$ \\
Reestudo & $1,8 \pm 0,6$ & $2,49 \pm 0,6$ & $<0,0001$ \\
Perda tardia & $1,7 \pm 0,32$ & $0,51 \pm 0,38$ & $<0,0001$ \\
Reestenose binária & $69(92 \%)$ & $3(12 \%)$ & $<0,0001$ \\
\hline
\end{tabular}


Raudales JC, et al. Modelo Experimental de Reestenose Intrastent em Artérias Coronárias de Suínos: Efeito do Implante de Stent Sobredimensionado. Rev Bras Cardiol Invasiva. 2010;18(1):55-61.

TABELA 2

Dados do ultrassom intracoronário

\begin{tabular}{lccc}
\hline & $\begin{array}{c}\text { Grupo A } \\
(\mathbf{n}=\mathbf{7 5})\end{array}$ & $\begin{array}{c}\text { Grupo B } \\
(\mathbf{n = 2 5 )}\end{array}$ & P \\
\hline Área luminal mínima* & & & 0,106 \\
$\quad$ Pré-stent & $27,4 \pm 4,3$ & $28,3 \pm 3,9$ & $<0,0001$ \\
Pós-stent & $40,7 \pm 0,3$ & $30,2 \pm 0,2$ & $<0,0001$ \\
$\quad$ Reestudo & $10,2 \pm 1,1$ & $19,5 \pm 1,1$ & $<0,0001$ \\
Volume de hiperplasia/mm de stent $\left(\mathrm{mm}^{3} / \mathrm{mm}\right)$ & $5,9 \pm 0,8$ & $1,8 \pm 0,7$ & \\
* Valores expressos como média \pm desvio padrão em $\mathrm{mm}^{2}$. & & \\
\hline
\end{tabular}
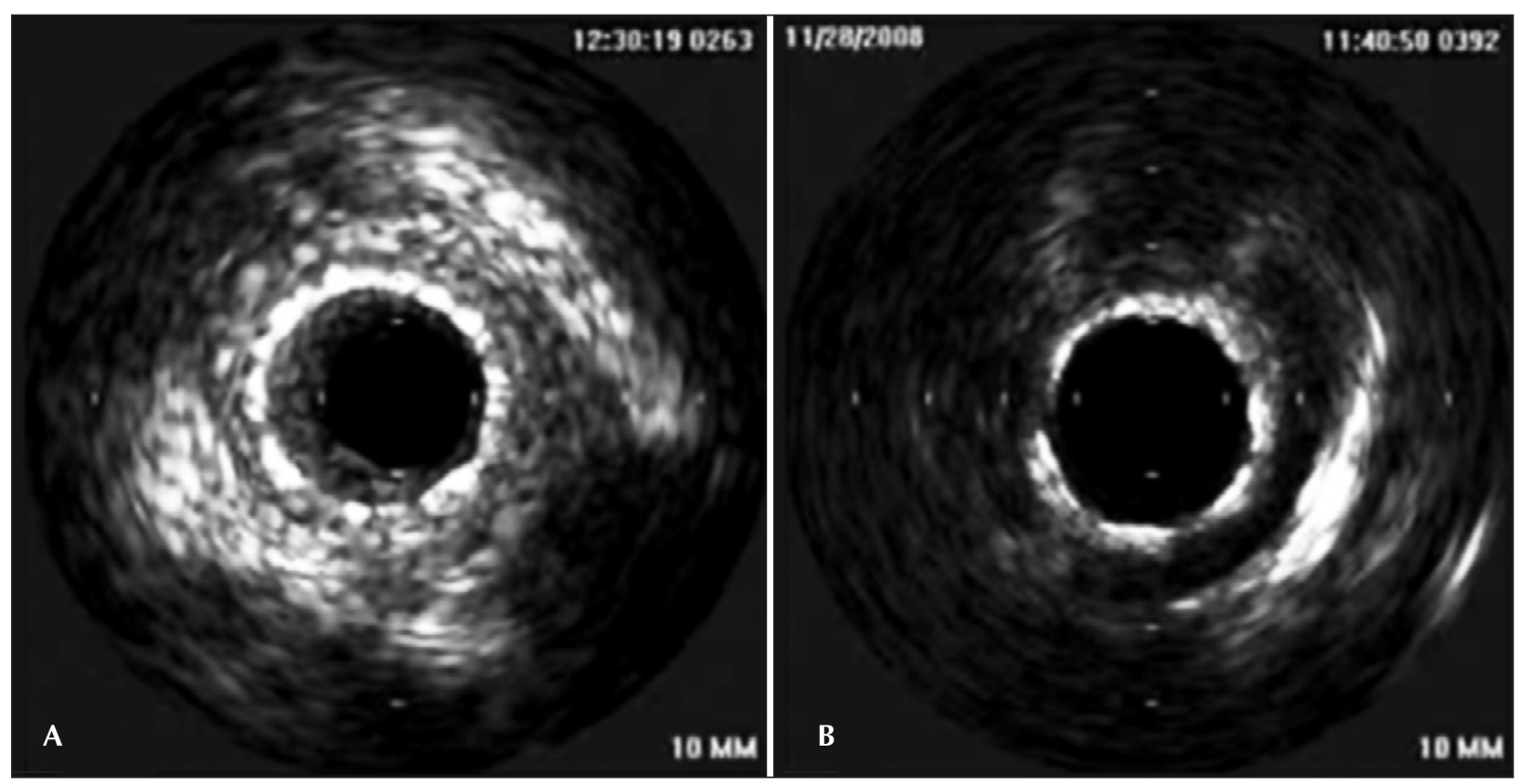

Figura 2 - Ultrassom intracoronário do reestudo em 28 dias demonstrando corte transversal de stent implantado no grupo stent sobredimensionado (A) e no grupo controle (B).

ção de matriz extracelular, contribuindo para a formação de neoíntima composta basicamente por células musculares lisas, monócitos e macrófagos. , $4,5-7,17,18^{2}$

A reestenose é conceituada como a resposta cicatricial exacerbada que se segue à lesão vascular ocasionada pela intervenção coronária percutânea, que, no caso dos stents, tem como mecanismo único a hiperplasia neointimal. 1,6-8,15,17,18,20 Também é relevante destacar que a reestenose intrastent apresenta características histológicas próprias, que a diferenciam da placa aterosclerótica convencional. ${ }^{20}$ A gravidade e a extensão da reestenose, avaliadas pela angiografia coronária quantitativa, podem ser classificadas de acordo com o padrão observado (focal, difusa, proliferativa e oclusiva), fornecendo informações importantes tanto de prognóstico quanto de orientação terapêutica. ${ }^{21}$
O modelo experimental de indução não-aterosclerótica de hiperproliferação neointimal aplicado neste estudo resultou em reestenose binária em 92\% dos suínos, similar à do modelo clássico de reestenose de Schwartz et al. ${ }^{5}$, que utilizaram angioplastia com balão modificado e sobredimensionado. Modelos posteriores de reestenose utilizando stents sobredimensionados também demonstraram resultados semelhantes. , $^{2,46-8,16-18}$ Schwartz et al. ${ }^{6,7}$ evidenciaram, em modelo suíno, que a proliferação neointimal é proporcional ao grau de lesão no endotélio vascular e que os mecanismos de reestenose ocasionada por balão ou por stent são diferentes. Deve-se salientar que o stent metálico não produz reação de corpo estranho, como inicialmente se imaginava, visto ter sido demonstrada ausência ou resposta celular inflamatória crônica mínima no local da lesão vascular ocasionada pelo stent. ${ }^{8,15}$ 
Schwartz et al. ${ }^{7}$ sugerem que essa excessiva hiperproliferação neointimal produzida pelo stent sobredimensionado decorra do fato de que cinco ou mais locais de lesão resultam em uma região localizada ao redor da circunferência do vaso, cada local com capacidade de gerar neoíntima. Esse tipo de padrão de lesão é muito diferente daquele observado com o uso do modelo de balão sobredimensionado, em que apenas um único ponto de dissecção é a resposta típica. Portanto, há dois fatores que favorecem a utilização do stent sobredimensionado como o melhor modelo para a criação de lesão vascular: o dano vascular pode ser classificado de acordo com um escore; ${ }^{2,7}$ e tanto o tamanho quanto a extensão exata da lesão podem ser mensurados e comparados diretamente com a resposta hiperproliferativa, utilizando métodos de regressão como sugerido por Bonan et al. ${ }^{2}$

A baixa taxa de reestenose intrastent de $12 \%$ no grupo controle em relação à dos stents convencionais em humanos pode em parte ser explicada pelo menor trauma tecidual vascular causado durante o implante de stent nesse grupo, visto que o diâmetro final do stent implantado era o mesmo do diâmetro de referência do vaso. Outro fator importante a considerar é a ausência de placa ateromatosa prévia no segmento tratado, a qual teoricamente deixa o tecido vascular menos suscetível a reação inflamatória pela trauma endotelial ocasionado pelo implante do stent.

A utilização do ultrassom intracoronário foi importante para assegurar a boa expansão do stent e a adequada aposição das hastes à parede do vaso. Também foi relevante para confirmar o diâmetro de referência do vaso e o diâmetro final do stent, certificando o cumprimento da metodologia proposta e os resultados obtidos neste estudo.

A taxa de mortalidade perioperatória, considerada aceitável pelos autores nessa fase inicial da curva de aprendizado da equipe multidisciplinar, pode ser reduzida. A septicemia, responsável por pouco mais da metade dos eventos, secundária à infecção subclínica por circovírus, pode ser controlada pela seleção rigorosa dos suínos e pela realização de quarentena prévia ao procedimento. A parada cardiorrespiratória durante indução anestésica, responsável por quase um terço dos eventos, foi atribuída à curva de aprendizado, pois ocorreu somente no início das atividades do grupo de pesquisadores. A ausência de eventos no pós-operatório foi decorrente do cuidadoso trabalho da equipe na prevenção de enfermidades e complicações.

Finalmente, a alta e significativa incidência de reestenose binária observada no grupo submetido a implante de stent sobredimensionado e a baixa taxa de reestenose binária obtida no grupo controle evidenciam a eficácia desse modelo experimental de indução de hiperproliferação neointimal, validando-o tanto para o estudo dos mecanismos fisiopatológicos da reestenose intrastent, quanto para as aplicações terapêuticas, como testes de novos fármacos, novos dispositivos e novos stents farmacológicos para prevenção e tratamento da reestenose intrastent.

\section{Limitações do estudo}

O modelo suíno pode diferir da reestenose humana em seus mecanismos fisiopatológicos; no entanto, o processo global e os resultados histopatológicos parecem ser idênticos àqueles encontrados na reestenose humana. A ausência de placa ateromatosa preexistente constitui outra limitação, visto que difere das condições reais em que o stent é implantado em humanos. A terceira limitação refere-se ao elevado custo desse modelo experimental, que requer unidade de hemodinâmica semelhante à utilizada em humanos, equipe multidisciplinar treinada e dispositivos de custo elevado, como stents e cateteres de ultrassom intracoronário.

\section{CONCLUSÃO}

O modelo experimental que consiste no implante intracoronário de stent sobredimensionado em suínos é eficaz na indução de proliferação neointimal intrastent exacerbada (reestenose). Esse modelo é de grande importância tanto para o estudo dos mecanismos fisiopatológicos da reestenose intrastent quanto para as aplicações terapêuticas, como testes de novos fármacos, novos dispositivos e novos stents farmacológicos, para prevenção e tratamento da reestenose intrastent.

\section{CONFLITO DE INTERESSES}

Os autores declararam inexistência de conflito de interesses relacionado a este manuscrito.

\section{REFERÊNCIAS}

1. Bienvenu JG, Tanguay JF, Theoret JF, Kumar A, Schaub RG, Merhi Y. Recombinant soluble P-selectin glycoprotein ligand1 -Ig reduces restenosis through inhibition of platelet-neuthrophil adhesion after doublé angioplasty in swine. Circulation. 2001; 103:1128-34.

2. Bonan R, Paiement P, Leung K. Swine model of coronary restenosis: effect of a second injury. Cathet Cardiovasc Diagn. 1996;38:44-9.

3. Gallo R, Padurean A, Toschi V, Bichler J, Fallon JT, Chesebo $\mathrm{JH}$, et al. Prolonged thrombin inhibition reduces restenosis after balloon angioplasty in porcine coronary arteries. Circulation. 1998;97:581-8.

4. Lowe HC, Schwartz RS, Mac Neill BD, Jang IK, Hayase M, Rogers C, et al. The porcine coronary model of in-stent restenosis: Current status in the era of drug-eluting stents. Catheter Cardiovasc Interv. 2003:60:515-23.

5. Schwartz RS, Murphy JG, Edwards WD, Camrud AR, Vliestra RE. Restenosis after balloon angioplasty: a practical proliferative model in porcine coronary arteries. Circulation. 1990;82:2190-200.

6. Schwartz RS, Huber KC, Murphy JG, Edwards WD, Camrud AR, Vliestra RE, et al. Restenosis and the proportional neointimal response to coronary artery injury: results in a porcine model. J Am Coll Cardiol. 1992;19:267-74. 
7. Schwartz RS, Kantor B, Holmes DR. The porcine model of coronary restenosis. In: Simon DI, Rogers C, editors. Vascular disease and injury. Preclinical research. New Jersey: Humana Press; 2001.

8. Alt E, Haehnel I, Beilharz C, Prietzel K, Peter K, Stemberg A, et al. Inhibition of neointima formation after experimental coronary artery stenting: a new biodegradable stent coating releasing hirudin and the prostacyclin analogue iloprost. Circulation. 2000;101:1453-8.

9. United States Food and Drug Administration 1994 Guidelines for the submission of research and marketing applications for interventional cardiology devices.

10. Colégio Brasileiro de Experimentação Animal. Princípios éticos na experimentação animal. São Paulo: COBEA; 1991.

11. Conselho Federal de Medicina Veterinária (CFMV). Resolução N. 714 de 20 de junho de 2002. Dispõe sobre procedimentos e métodos de eutanásia em animais, e dá outras providencias. [citado 22 mar 2010]. Disponível em: http://www.funed. mg.gov.br/comissao-de-etica-no-uso-de-animais/resolucao_ 714.pdf.

12. Gallo R, Padurean A, Jayaraman T, Marx S, Roque M, Adelman $S$, et al. Inhibition of intimal thickening after balloon angioplasty in porcine coronary arteries by targeting regulators of the cell cycle. Circulation. 1999;99:2164-70.

13. Raudales JC, Zago AJ. Uso de stents coronarianos recobertos de heparina em pacientes de alto risco [tese]. Porto Alegre: Faculdade de Medicina, Universidade Federal do Rio Grande do Sul; 2000.

14. Roubin GS, Califf RM, O'Neill WW, Phillips HR, Stack RS, editors. Interventional cardiovascular medicine. Principles and practice. New York (NY): Churchill Livingstone; 1994.

15. Gertz SD, Fallon JT, Gallo R, Taubman MB, Banai S, Barry $W L$, et al. Hirudin reduces tissue factor expression in neointima after balloon injury in rabbit femoral and porcine coronary arteries. Circulation. 1998;98:580-7.

16. McKenna CJ, Burke SE, Opgenorth, Padley RJ, Camrud AR, Jonson J, et al. Selective $\mathrm{ET}_{\mathrm{A}}$ receptor antagonism reduces neointimal hyperplasia in a porcine coronary artery stent model. Circulation. 1998;97:2551-6.

17. Frimerman A, Welch PJ, Jin X, Eigler N, Yei S, Forrester J, et al. Chimeric DNA-RNA hammerhead ribozyme to proliferating cell nuclear antigen reduces stent-induced stenosis in a porcine coronary model. Circulation. 1999;99:697-703.

18. Suzuki T, Kopia G, Hayashi S, Bailey LR, Llanos G, Wilensky $\mathrm{R}$, et al. Stent-based delivery of sirolimus reduces neointimal formation in a porcine coronary model. Circulation. 2001; 104:1188-93.

19. Carter AJ. Atherosclerotic porcine coronary stent model. Technical methods for preclinical studies. In: Simon DI, Rogers C, editors. Vascular disease and injury. Preclinical research. New Jersey: Humana Press; 2001.

20. Raudales JC, Zago AC, Zago AJ, Campos MR, Casco MF, Wachleski J. Placa coronária aterosclerótica vulnerável: estado atual. Rev Bras Cardiol Invasiva. 2006;14:314-23.

21. Mehran R, Dangas S, Abizaid A, Mintz GS, Lansky AJ, Satler $L F$, et al. Angiographic patterns on in-stent restenosis: classification and implications for long-term outcome. Circulation. 1999;100:1872-8. 